\title{
Evaluación del beneficio de la letra C artículo 14 ter de la Ley sobre Impuestos a la Renta
}

\section{Assessment of the benefit of the letter C, article 14 ter of the income tax law}

\author{
Germán R. Pinto Perry ${ }^{1}$
}

\begin{abstract}
RESUMEN
La presente investigación establece los efectos en la carga tributaria total (Impuesto a la Renta de Primera Categoría más el Impuesto Global Complementario) que afectan a las contribuyentes pymes que acogen las normas de la letra $C$ del artículo 14 ter de la Ley sobre Impuestos a la Renta, estableciendo la naturaleza de las diferencias cuando se trata de contribuyentes acogidos a la letra A del artículo 14 (14 A) o letra B del mismo articulado (14 B) ambos de la Ley sobre Impuestos a la Renta, haciendo una reflexión de cómo la teoría económica establece procedimientos en la determinación del resultado tributario que deben resultar atractivo para determinados contribuyentes.

Se utilizó una metodología según el paradigma cualitativo, pues se espera cavilar de la naturaleza de las diferencias, más allá de su medición. Se realizó una investigación descriptiva y utilizó una herramienta de investigación de "microsimulación tributaria" que permitió obtener resultados según la manipulación de la variable "retiros" que fue modificada según un porcentaje de la utilidad afecta a impuestos. Los objetivos planteados evalúan los efectos en la carga tributaria total, entendida como la suma del IRPC y el IGC (no se considera el impuesto adicional), esperando determinar un menor impuesto comparativamente en relación con la opción de no impetrar el beneficio, como también la evaluación de la carga tributaria entre contribuyentes acogidos a la letra A y a la letra B del artículo 14 de la ley del ramo.

Los resultados obtenidos permiten afirmar que el legislador estableció un incentivo que resulta pernicioso para los contribuyentes 14 B en relación con los 14 A.
\end{abstract}

Palabras clave: Beneficio Tributario - Microsimulación - Pymes

Recepción: 13/06/2018. Aprobación: 12/1/2018.

1 Profesor del Departamento de Contabilidad y Auditoría de la Facultad de Administración y Economía de la Universidad de Santiago, Santiago, Chile. german.pinto@usach.cl 


\begin{abstract}
ABSTRAC
The present investigation establishes the effects on the total tax burden (First Category Income Tax + Complementary Global Tax) generated by SME taxpayers that accept the rules of letter $\mathrm{C}$ of article 14 ter of the Law on Income Taxes, establishing the nature of the differences between letter A of article 14 (14 A) or letter B of the same article (14 B) both of the Law on Income Taxes.

The qualitative paradigm methodology was used, becasuse it is expected to reflect on the nature of the differences, instead of their measurement. The investigation was describe and use a research tool of "tax microsimulation" that allowed obtaining results according to the manipulation of the variable "withdrawals" as a percentage of the tax utility.

Methodology was used according to the qualitative paradigm, because we want to reflect on the nature of the differences, beyond their measurement. A descriptive investigation was carried out and a research tool of "tax microsimulation" was used to obtain results according to the manipulation of the variable "withdrawals" as a percentage of the taxable profit. The proposed objectives evaluate the effects on the total tax burden, understood as the sum of the IRPC and the IGC (the additional tax is not considered), hoping to determine a lower tax comparatively in relation to the general regime, as well as the evaluation of the burden tributary among taxpayers under letter A and letter B of article 14 of the law of the branch.

The results obtained allow to affirm that the legislator established an incentive that is pernicious for the taxpayers $14 \mathrm{~B}$ in relation to the $14 \mathrm{~A}$.
\end{abstract}

Key Word: Tax Benefict - Tax Microsimulation - Sme Taxpayers

\title{
INTRODUCCIÓN
}

La presente investigación evalúa el beneficio tributario contenido en la letra C del artículo 14 ter de la Ley sobre Impuestos a la Renta (LIR) que concede una rebaja en Renta Líquida Imponible (RLI) equivalente al 50\% de las utilidades no retiradas de la empresa durante un ejercicio comercial. Este beneficio puede ser impetrado por los contribuyentes acogidos a la letra A del artículo 14 de la LIR (renta atribuida) y también a los acogidos a la letra B del mismo articulado (sistema parcialmente integrado) siempre que sus ingresos no superen en promedio anual de ingresos de las 100.000 unidades de fomento (UF) en los últimos tres años comerciales. Este beneficio genera una diferencia en la carga tributaria total (CTT) entre los contribuyentes acogidos a la letra B del artículo 14 (en adelante: 14B), siendo ésta más elevada que la generada por los contribuyentes acogidos a la letra A del artículo 14 (en adelante: 14A), resultando esto una evidente discriminación innecesaria.

La Ley sobre Impuestos a la Renta (LIR) identifica este beneficio (el título ocupado en el texto de la ley) como "Incentivo al ahorro para medianas y pequeñas empresas" representando de esta forma, un tipo de "beneficio tributario" que se establece cuando la autoridad fiscal quiere intervenir en la economía. Musgrave y Musgrave (1992, p256) así lo ha interpretado e ilustrado tomando el esquema del "flujo circular de la economía" de Samuelson (1966) ilustrando cómo los impuestos inhiben una conducta, como en caso de los gastos rechazados; e incentivan acciones a través de beneficios, como la depreciación acelerada y el no gravar las utilidades que son reinvertidas. Otros 
autores también han demostrado que los tributos² afectan a la economía de un país (Grahan, Hanlon y Shevlin, 2011), como también su carácter distorsionador en los mercados del ahorro (Jacagos y Bovenberg, 2009) y laboral (Otorna, Ottone, Ponzano y Scacciati, 2008).

Pese a esa influencia positiva o negativa, los tributos son la "fuente más importante de ingreso para el gobierno" (Larraín,2011; p 70), y por lo tal es utilizada reiteradamente para aumentar la recaudación fiscal, como también para incentivar y corregir conductas, siendo, incluso, consideradas con imperfecciones, y como un mal necesario (Holmes y Sunstein, 2011).

Autores como Haufler (1997), Crocker y Slemrod (2005), Devereux y Griffth (2002) y en Australia Hanlon y Pinder (2006) evidencian los efectos distorsionadores que tienen los tributos en la economía de un país. Esto justifica la necesaria intervención que tuvo que realizar el legislador para evitar efectos negativos producidos por el alza en la tributación de los contribuyentes del Impuesto a la Renta de Primera Categoría (IRPC) que llevan contabilidad completa ${ }^{3}$ que se acogieron a las normas del artículo 14 A o 14 B de la LIR, aumentando la tasa de este tributo a un 25\% y un $27 \%$ respectivamente 4 .

Tal como se evidenció en los trámites constitucionales de formación de las leyes que materializaron las Reformas Tributarias de los años 2014 y 2016, el tema fue controvertido y fue necesario un protocolo de acción cuando el proyecto de ley ${ }^{5}$ ingresó al Senado, siendo imperiosa una reunión fuera del Congreso para morigerar las iniciales ideas legislativas que aplicaban el régimen de renta atribuida a todos los contribuyentes, lo que perjudicaría la inversión y el ahorro de las empresas. Por otro lado, tal como afirma Agostini (2017) $)^{6}$ tener dos regímenes de tributación, el 14 A y 14 B, genera una complicación innecesaria que solo redunda en ineficiencias.

En este concierto de evaluaciones políticas y técnicas, surge la norma contenida en la letra C del artículo 14 ter de la LIR que concede a los contribuyentes del IRPC que llevan contabilidad completa acogidos a las normas del artículo 14 A o 14 B, cuyos ingresos promedios de los últimos 3 años comerciales, no superen las 100.000 UF, puedan rebajar de la Renta Líquida Imponible $(\mathrm{RLI})^{7}$ las utilidades no retiradas. Este beneficio está dirigido a las empresas de mediano y pequeño tamaño, las cuales tienen ingresos no superiores al monto en UF que ya se mencionó.

Las utilidades no retiradas se estiman considerando la RLI menos los retiros efectivos realizados durante el ejercicio. De esta suma se toma solo el 50\% que sería el monto a rebajar de la RLI, permitiendo de esta forma que no paguen el IRPC las utilidades que aún no han sido retiradas.

Existe una restricción adicional para los contribuyentes acogidos a las normas de la letra B del

2 Tributo es el término general que se ocupa para referirse a impuestos, contribuciones y tasas.

3 Nos referiremos en adelante a estos sujetos como "empresas".

4 Los contribuyentes 14B se afectaron con una tasa provisoria de 25,5\% por el ejercicio comercial 2017, para aplicar la del

$27 \%$ en los futuros años.

5 De la primera reforma materializada en la ley 20.780.

6 Se hace presente que la fuente de esta opinión no es "científica" pues corresponde solo a una publicación periodística.

Sin embargo, se incluye en este trabajo solo para ilustrar la preocupación profesional que está en el problema de investigación tratado.

7 La RLI es la utilidad afecta al IRPC que se determina según lo indicado por el legislador en los artículos 29 al 33 en concordancia del artículo 21 y 41 de la LIR. 
artículo 14, pues deben agregar a la RLI del año siguiente de aquel en que realizaron la rebaja que comentamos, una cantidad equivalente al 50\% de los retiros efectivos del periodo en curso hasta completar el monto del beneficio aplicado año tras año. La mecánica se explica con el siguiente ejemplo numérico:

\section{Antecedentes}

RLI al final del año 1, \$100.000.000

Retiros efectivos del año 1, \$30.000.000

RLI al final del año 2, \$150.000.000

Retiros efectivos del año 2, \$50.000.000

\section{Cálculos del Año 1}

\section{DETALLE}

$R L I$

Menos: $50 \%$ utilidades no retiradas

RLI inicial

Menos: retiros efectivos

Utilidades no retiradas

$50 \%$ a considerar

RLI afecta a IRPC

IRPC $25 \%$ y $27 \%$ $\begin{array}{ll}\text { ART. 14 A } & \text { ART. 14 B } \\ 100.000 .000 & 100.000 .000\end{array}$

100.000.000

$\underline{(30.000 .000)}$

70.000 .000

35.000 .000

$\underline{(35.000 .000)} \quad \underline{(35.000 .000)}$

$65.000 .000 \quad 65.000 .000$

$16.250 .000 \quad 17.550 .000$

\section{Cálculos del Año 2}

\section{DETALLE}

$R L I$

Menos: 50\% utilidades no retiradas

RLI inicial

Menos: retiros efectivos

Utilidades no retiradas

$50 \%$ a considerar

Más: $50 \%$ retiros efectivos, solo $14 \mathrm{~B}$

RLI afecta a IRPC

IRPC $25 \%$ y $27 \%$

\section{ART.14A ART.14B \\ $150.000 .000 \quad 150.000 .000$}

150.000 .000

$\underline{(50.000 .000)}$

100.000.000

50.000 .000

$(50.000 .000) \quad(50.000 .000)$

\begin{tabular}{rr}
25.000 .000 \\
\hline $\mathbf{1 0 0 . 0 0 0 . 0 0 0}$ & $\mathbf{1 2 5 . 0 0 0 . 0 0 0}$
\end{tabular}

$25.000 .000 \quad 33.750 .000$

Podemos apreciar claramente cómo los contribuyentes del artículo 14 B tienen una mayor carga tributaria, tanto por la mayor tasa como también por el agregado que tienen que hacer para "devolver" el beneficio impetrado en el año anterior. 
La presente investigación tiene por objetivo general el evaluar los efectos en la carga tributaria total de los contribuyentes acogidos a la letra B del artículo 14 cuando impetran el beneficio otorgado por la letra C del artículo 14 ter de la LIR, estableciendo su comparación con el efecto en la carga tributaria total de los contribuyentes acogidos a la letra A del artículo 14 de la LIR.

Como objetivos específicos se determinará las variables que determinan la mayor o menor carga tributaria total en los contribuyentes en estudio.

También se establecerá la naturaleza de las diferencias determinadas entre los contribuyentes de las letras A y B del artículo 14 de la LIR.

Esta investigación plantea la siguiente conjetura: El Legislador ha generado una aplicación discrecional del beneficio contenido en la letra C del artículo 14 ter de la LIR, que perjudica a los contribuyentes acogidos a la letra B del artículo 14 en relación con los acogidos a la letra A del artículo 14 de la LIR, dado que la aplicación de este beneficio reditúa distintos efectos a contribuyentes que tienen igual patrimonio.

\section{METODOLOGÍA, MATERIALES Y MÉTODO}

La metodología utilizada fue un enfoque cualitativo porque el interés de la investigación es reflexionar sobre las diferencias en las cargas tributarias totales que determinan los contribuyentes acogidos a las letras A o B del artículo 14 de la LIR y que hayan impetrado el beneficio de la letra C del artículo 14 ter del mismo cuerpo legal, más allá de establecer la cuantía de esas discrepancias, siendo los números un atributo o cualidad más que un resultado, característica fundamental de las investigaciones bajo el paradigma cualitativo (Taylor y Bogdan, 1987)

El método utilizado corresponde a una investigación del tipo descriptiva, pues tal como señala Hernández, Fernández y Baptista (2010, p 80) se pretende especificar propiedades, características y rasgos importantes de cualquier fenómeno que se analice. Esto es importante porque se señalarán los efectos que genera la norma en estudio.

La herramienta de investigación es la "micro simulación tributaria", herramienta utilizada en el estudio de los efectos derivados de las políticas públicas internacionales (Atkinson, Bourguignon, O’Donoghue, Sutherland y Utili, 2002) como también en las nacionales (Mardones, 2010).

Tal como señala Tarifa (2005), los modelos en este tipo de estudios permiten abstraer datos de la realidad, manipularlos y obtener resultados de situaciones que no pueden ser experimentadas en la actualidad, ya sea por lo difícil de utilizar los datos por lo difícil de acceder al sujeto de estudio, como también porque los experimentos pueden ser económicamente caros o el sistema evoluciona a una velocidad que es difícil para el investigador (Duncan,2002).

La investigación se basó en la aplicación del modelo de microsimulación tributaria diseñado según las normas legales contenidas en la letra C del artículo 14 ter de la LIR según esquema 


\section{Esquema 1. Mecanismo de Aplicación del Beneficio de la Letra C del Artículo 14 ter de la LIR}

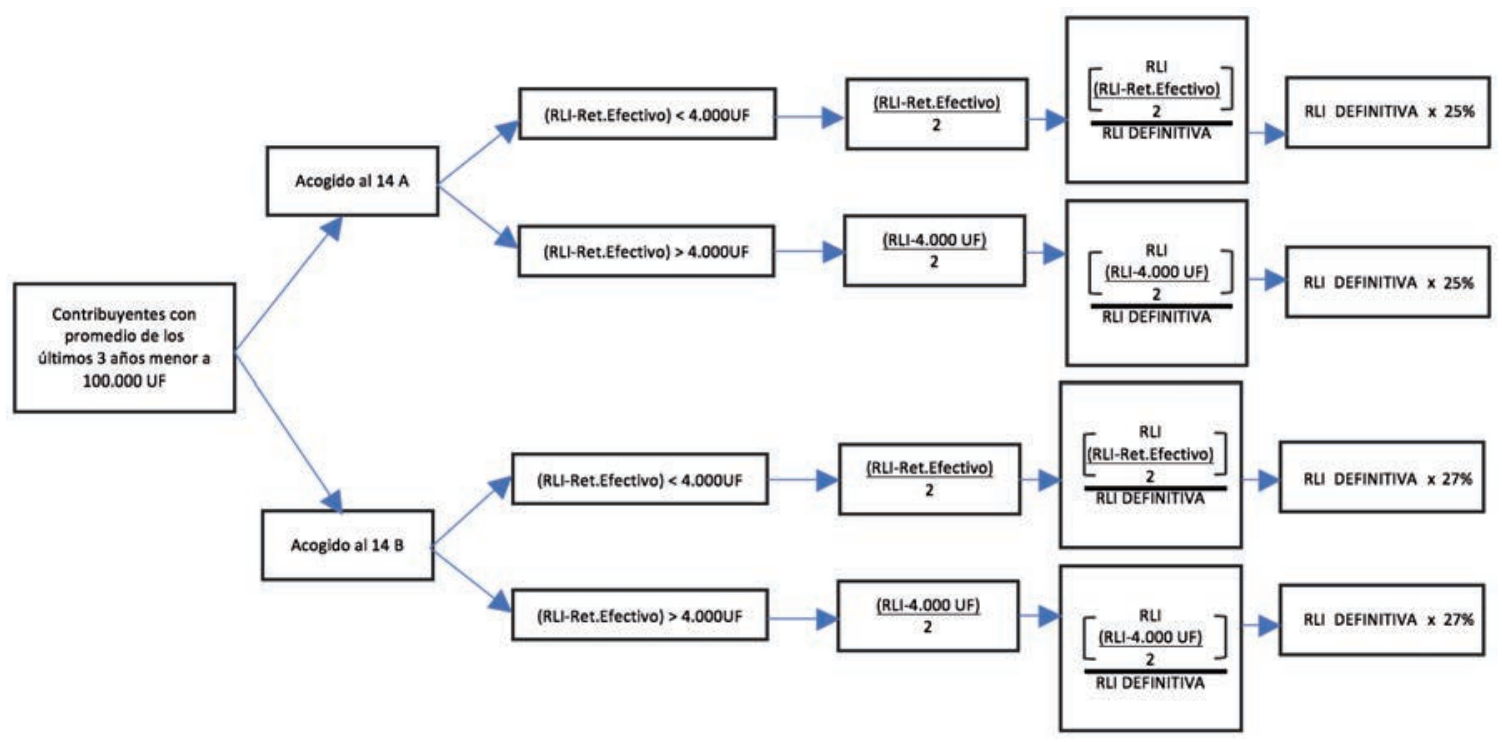

Fuente: Elaboración Propia.

Lo anterior evidencia la aplicación de la normativa según el criterio general y que no distingue entre contribuyentes 14 A o 14 B, salvo por el hecho de que los primeros tienen una tasa del Impuesto a la Renta de Primera Categoría (IRPC) del 25\% y los segundos una de $27 \%$, siendo esto la evidente diferencia en la carga tributaria total, sin considerar qué pasa cuando se considera el Impuesto Global Complementario (IGC), lo cual será considerado el siguiente esquema.

\section{Esquema 2: Aplicación del Beneficio en la RLI}
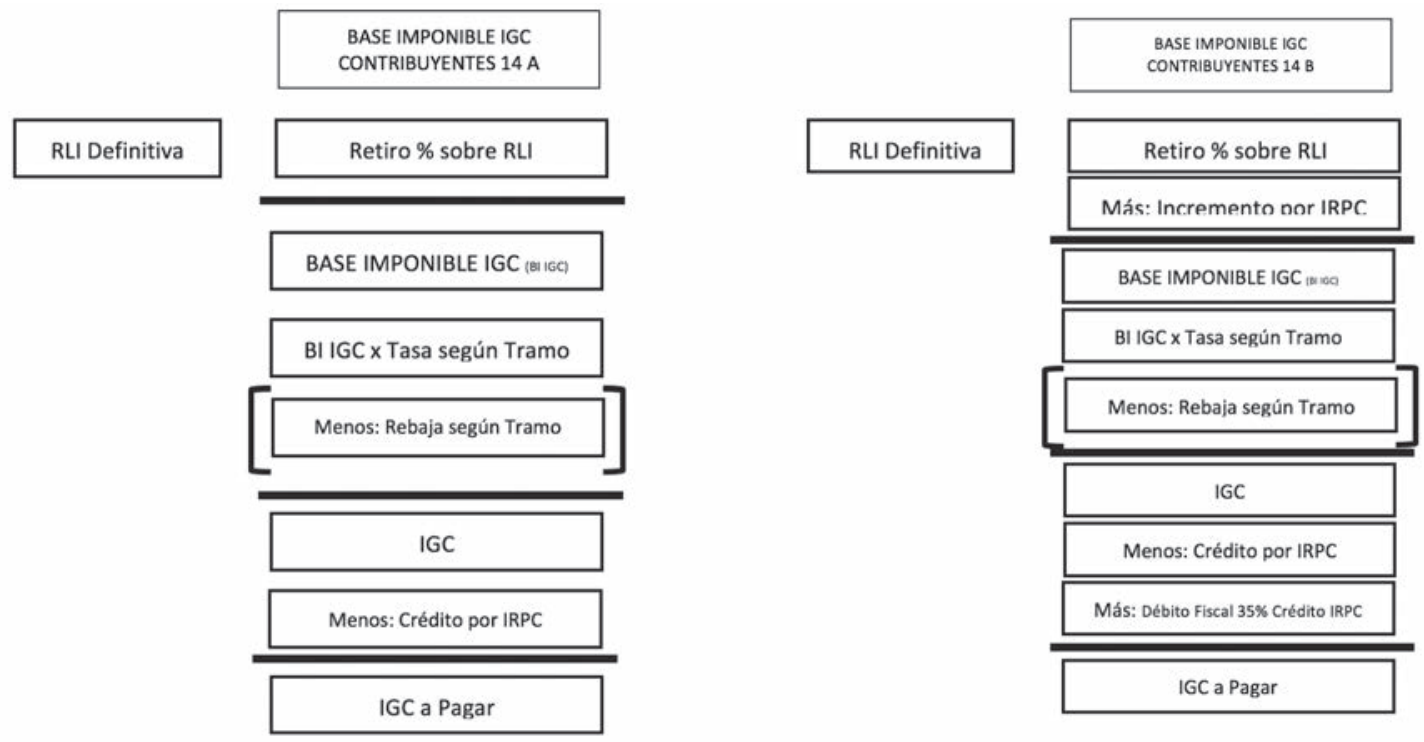
El concepto de "carga tributaria total" (CTT) con la cual trabajará esta investigación, es el resultado de sumar el IRPC que paga la empresa con el IGC que debe pagar el socio o accionistas. Para efectos de simplificación se consideró un solo dueño.

Es importante señalar que la CTT puede resultar un monto positivo o uno negativo dependiendo del monto del IRPC en relación con el IGC. Si el IRPC es mayor que el IGC determinado se genera una CTT negativa porque se devuelve el IRPC que se pagó por sobre el IGC. Si es positiva, el análisis es el inverso.

El análisis sigue, pues el articulado que estudia esta investigación señala que solo los contribuyentes del 14 B están obligados a devolver al año siguiendo de aquel en que hubieran impetrado el beneficio, el 50\% de los retiros efectivos realizados en ese segundo año hasta completar el monto del beneficio total utilizado en el ejercicio en curso como en los anteriores. Esto genera el siguiente esquema solo aplicable a los contribuyentes acogidos a la letra B del artículo 14 de la LIR.

\section{Esquema 3. Devolución del Beneficio al Año Siguiente y Sucesivos de Haberlo Impetrado}
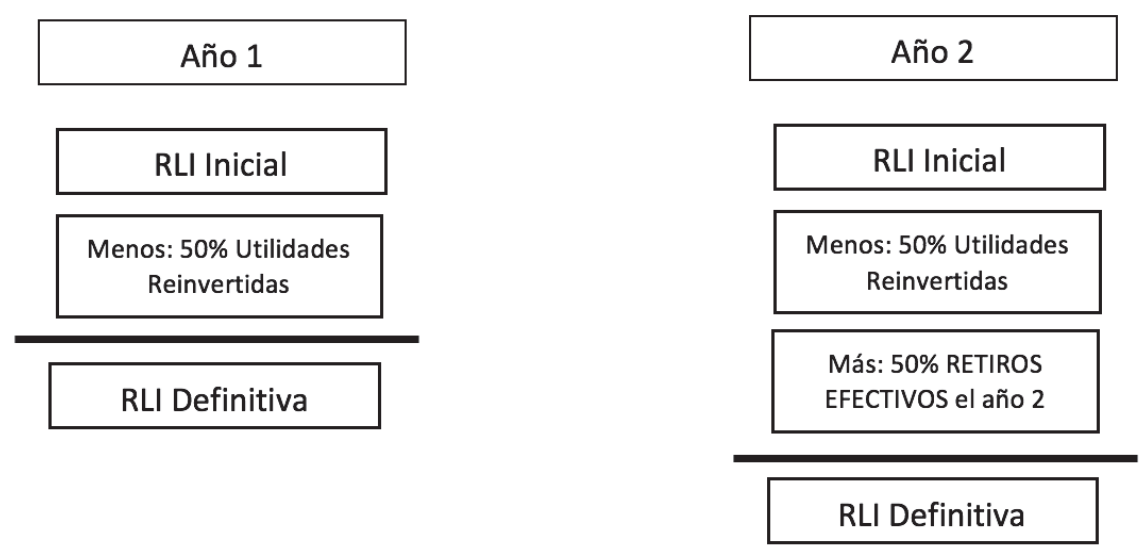

Fuente: Elaboración Propia.

El Año 2 ilustra el tratamiento señalado en la norma legal, sumando el 50\% de los retiros efectivos de ese periodo generando, obviamente, una mayor base imponible que de no efectuar retiros.

\section{RESULTADOS}

Una vez aplicada la microsimulación tributara presentada anteriormente, se obtuvieron los siguientes resultados.

Se realizaron ejercicios de microsimulación de un contribuyente prototipo que cumple los requisitos para impetrar el beneficio de la letra C del artículo 14 de la LIR acogido tanto a la letra A como a la letra B del artículo 14 de la LIR, tomando como variable de estudio el monto de los 
retiros. Esta variable se analizó considerando la cantidad retirada aumentando desde el 10\% hasta el $100 \%$ de la RLI de un ejercicio cualquiera.

\section{Gráfico 1: Análisis del IRPC con Retiros cuyo Monto Varía entre el 10\% al 100\% de la RLI}

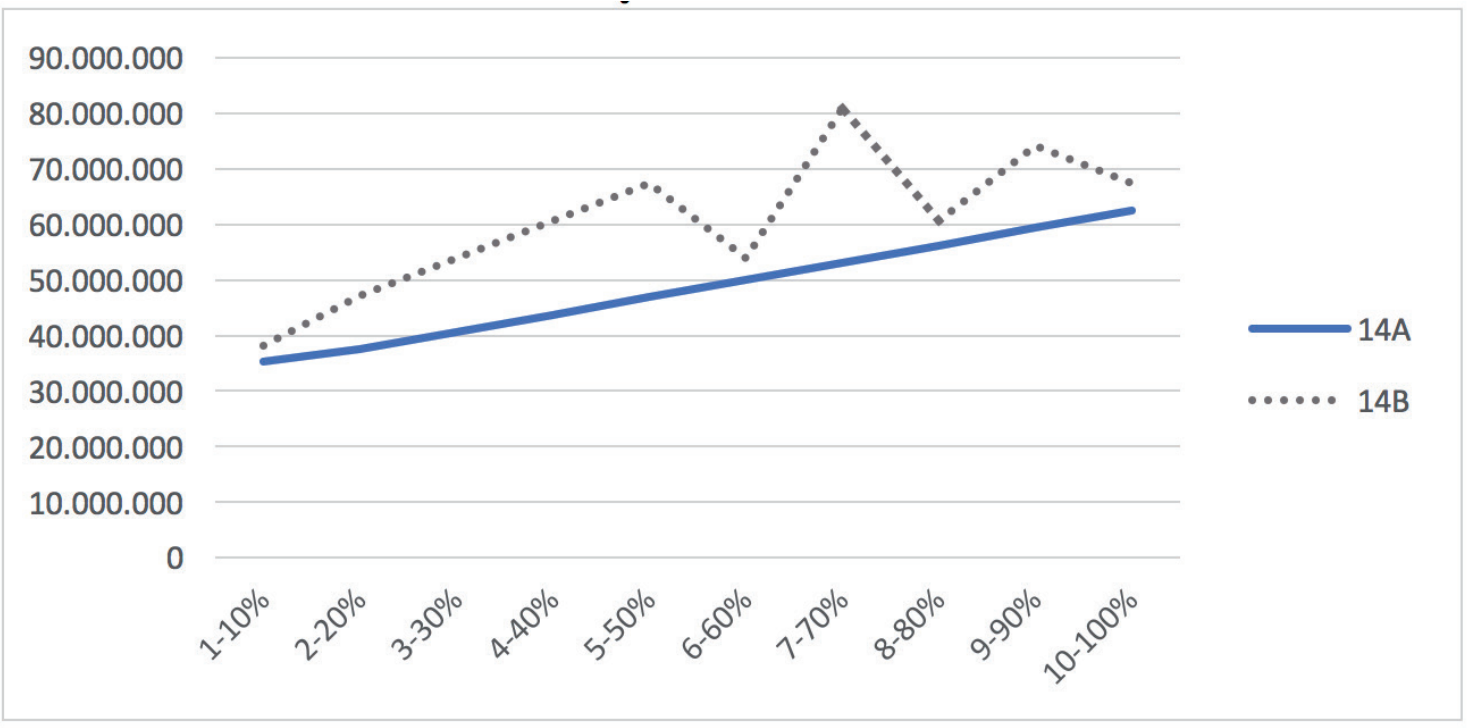

Fuente: Elaboración propia con base a los resultados.

Este gráfico ilustra el comportamiento de el monto del IRPC pagado por un contribuyente acogido a las normas de la letra A del artículo 14 de la LIR, y otro contribuyente con las mismas características acogido a las normas de la letra B del mismo articulado. Se puede apreciar claramente que la carga de los contribuyentes del 14 B es mayor en todos los casos, hecho evidente pues la tasa de estos contribuyentes es $27 \%$ en ves del $25 \%$ que afecta a los contribuyentes 14 A. Este hecho no tiene ninguna sorpresa, situación que ya fue estudiada en otra investigación. Sin embargo, resulta particularmente curioso el comportamiento de la curva de los contribuyentes 14 B cuando se retira el $60 \%$ de la RLI donde la carga tributaria es menor que en porcentajes menores de este hecho de retiros cuando analicemos el comportamiento particular de este contribuyente. 


\section{Gráfico 2: IGC determinado de Acuerdo al Retiro del 10\% al 100\% de la RLI}

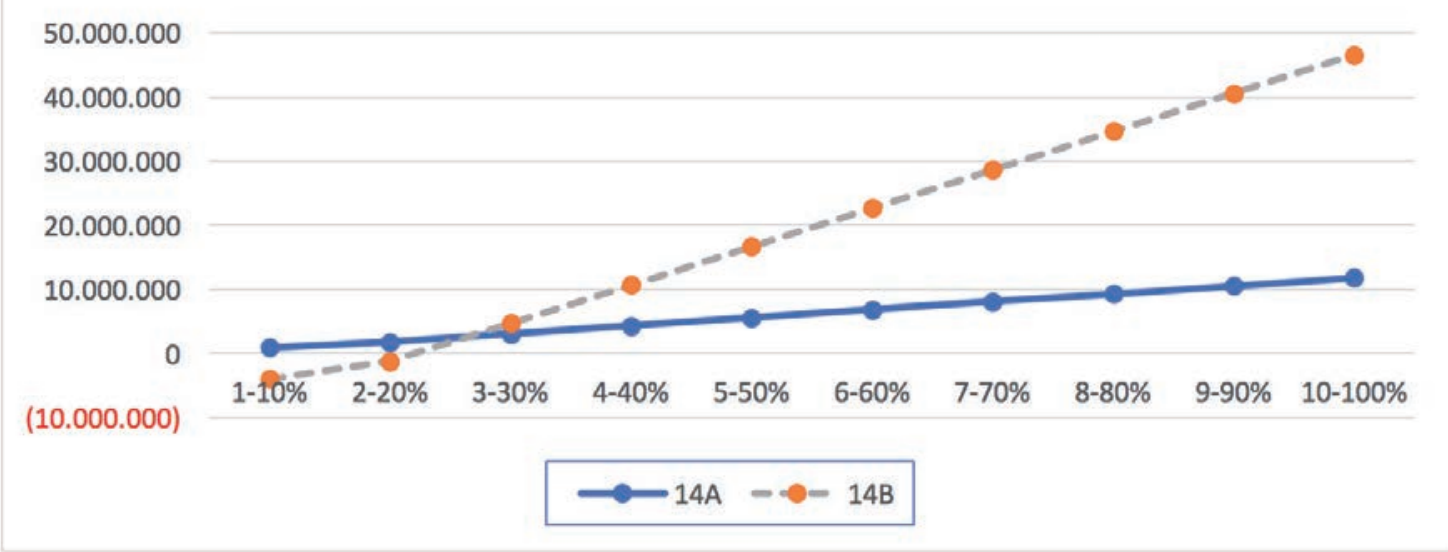

Fuente: Elaboración propia con base a los resultados.

Distinta es la situación cuando analizamos la carga tributaria del IGC, pues de retirar entre el $10 \%$ y el 30\% de la RLI, los contribuyentes $14 \mathrm{~B}$ se ven beneficiados en relación con los 14A, hecho que cambia abruptamente a contar de retirar $40 \%$ y hasta el $100 \%$.

Este hecho es claramente explicable porque los contribuyentes del 14B solo tributan con sus IGC cuando realizan retiros, en cambio los contribuyentes del 14A afectan su IGC por la RLI atribuida y de retirar una cantidad menor a la RLI, no les genera una mayor carga tributaria. Por otro lado, los contribuyentes 14B tienen una mayor carga tributaria en su IGC porque tienen que devolver el 35\% del crédito por el IRPC que consideraron en su declaración del tributo personal, siendo esto una evidente rémora en la rentabilidad comparativa de estos contribuyentes. Ahora bien, podemos apreciar que el beneficio de la letra $C$ del artículo 14 ter que estudiamos, no afecta a la tributación del IGC.

\section{Gráfico 3. CTT Determinada Mediante Retiros del 10\% al 100\% de la RLI}

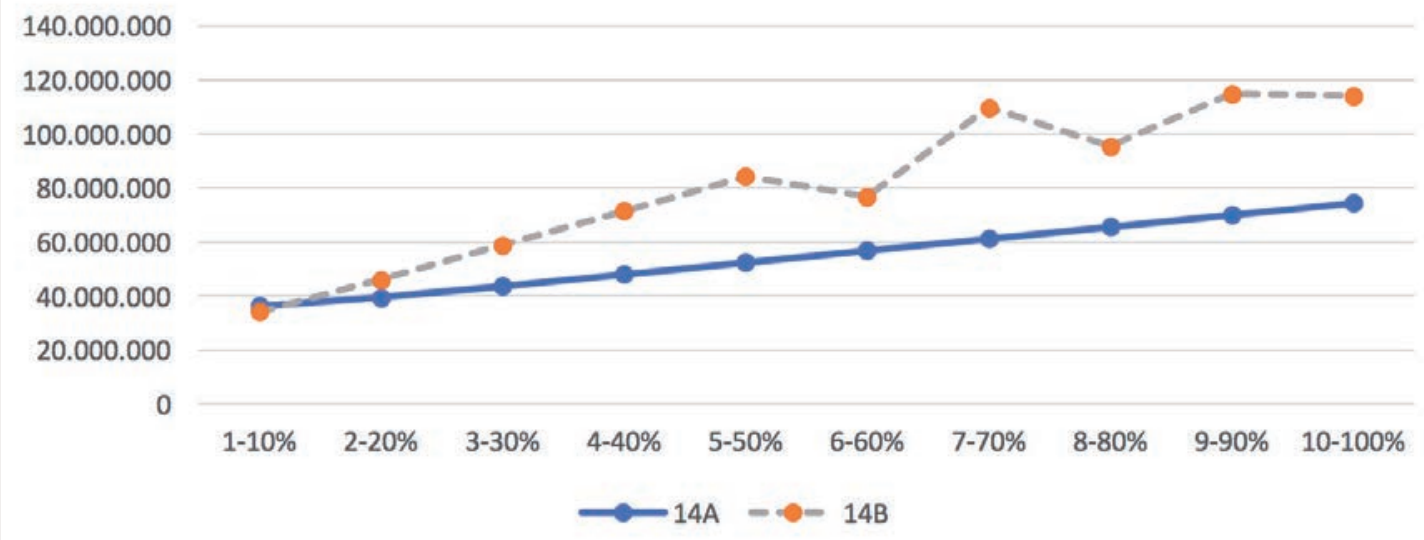

Fuente: Elaboración propia con base a los resultados. 
El análisis tiene que ser completado con el estudio de la CTT que, como ya se indicó, suma la tributación del IRPC con la del IGC. Esto arroja la evidencia que el artículo 14B es más caro, incluso perdiendo la situación beneficiosa evidenciada en el Gráfico 2 que ilustraba que con retirar el $30 \%$ de la RLI el IGC era más bajo que un 14A. Esto hecho se ve disminuido pues con los mismos niveles de retiro, la CTT solo es más baja en un 14B cuando se retira el 10\% de la RLI según la carga tributaria de un contribuyente $14 \mathrm{~A}$ en iguales condiciones.

A continuación, se presentarán los estudios analizando en forma aislada a los contribuyentes del artículo 14B, pues ellos tienen una rémora adicional en su CTT, que es la obligación de devolver el 50\% de los retiros efectivos del año o años siguientes en que se impetró el beneficio en estudio. Para ello, se realizó una microsimulación manteniendo un porcentaje fijo de retiros, es decir, todos los años de retiró el 10\% de la RLI, y se analizó el comportamiento en 5 años, manteniendo estables los niveles de RLI. Se utilizó este horizonte temporal (5 años) porque la norma fija en ese lapso el periodo mínimo de permanencia para estar acogido a las normas 14A o 14B.

\section{Gráfico 4, IRPC Determinado con Retiros de acuerdo al 10\% al 50\% de la RLI}

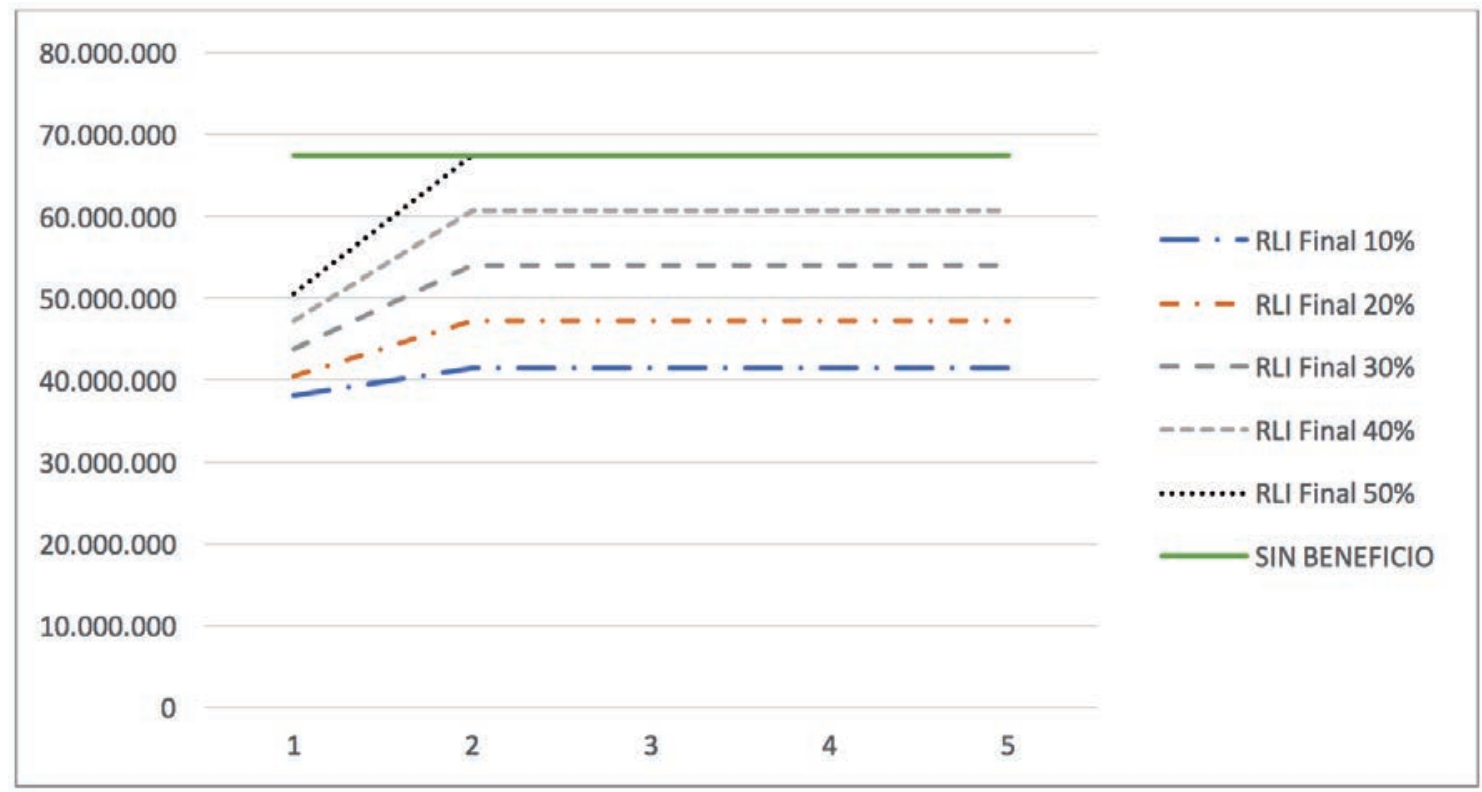

Fuente: Elaboración propia con base a los resultados.

Este gráfico muestra una situación evidente: que la CTT es mayor cuando no hay beneficio. Evidentemente, estamos en una situación donde el contribuyente ha optado por no retirar sus utilidades, sino capitalizarlas, objetivo buscado por el legislador.

Otro aspecto importante que se evidencia es que, fruto de la devolución del $50 \%$ de los retiros efectivos de cada año, el beneficio se neutraliza y queda en una constante. 


\section{Gráfico 5, IRPC Determinado por Retiro del $60 \%$ al $90 \%$ de la RLI}

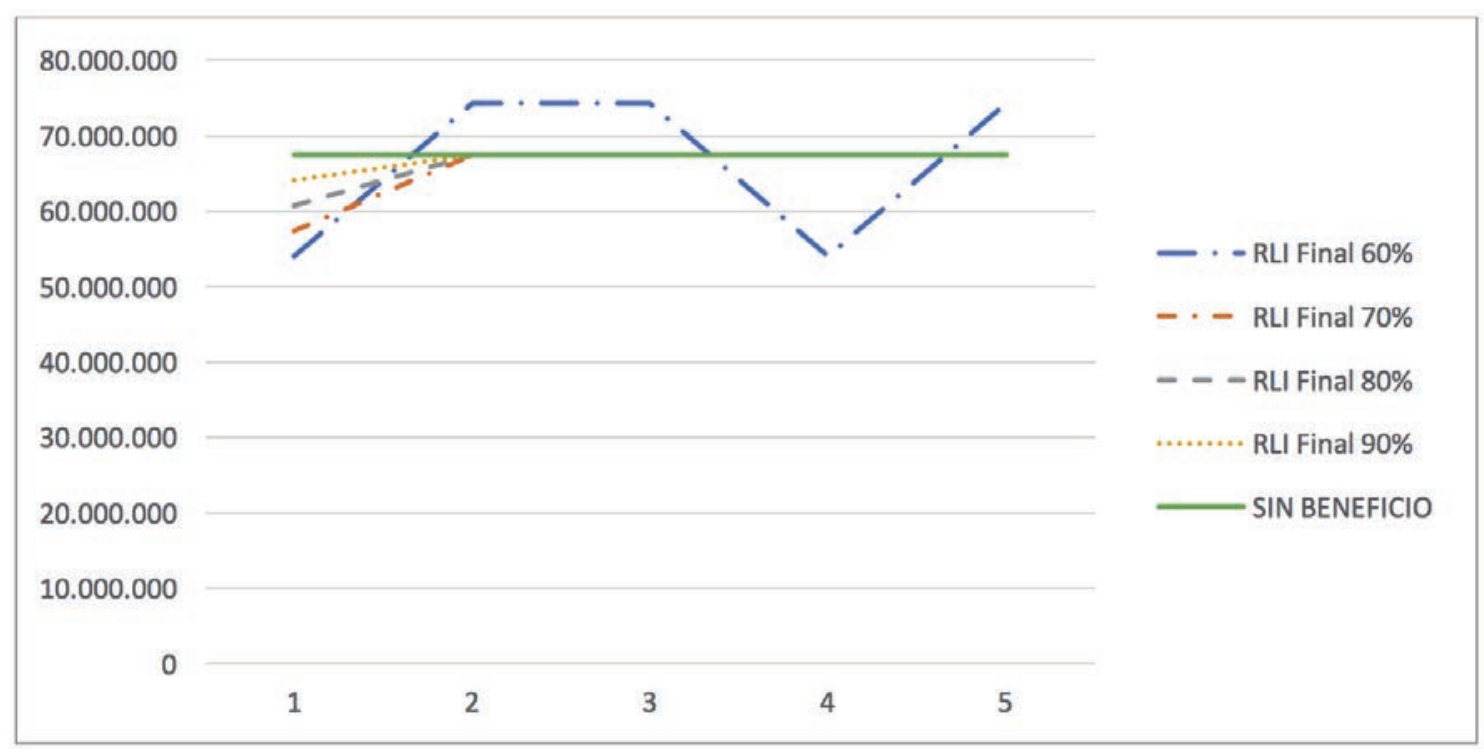

Fuente: Elaboración propia con base a los resultados.

Este gráfico evidencia un hecho particular: a medida que aumentan los retiros, la brecha a contar del segundo año se acorta llegando igualarse cuando se retira cada año el 50\% de la RLI. Se unen las curvas porque el rebajar el 50\% de las utilidades no retiras, se neutraliza con sumar el $50 \%$ de los retiros efectivo de los años siguientes.

Constatamos una situación particular. Cuando se retira el 60\% de la RLI se genera un comportamiento dispar en la curva de la CTT pues el IGC aumenta cuando hay muchos retiros, hecho evidente, y también la RLI aumenta porque el incrementar la utilidad con el 50\% de los retiros efectivos del año, puede generar una suma mayor que la obtenida de no impetrar el beneficio en estudio. Sin duda que este hecho solo puede ser descubierto con el análisis y simulación de los efectos de la normativa.

Con retiros superiores al 60\% se iguala la CTT a la situación de no haber impetrado el beneficio, hecho evidente, porque el propósito del legislador es incentivar a que los contribuyentes no realicen retiros. Sin embargo, también nos permite afirmar que el retirar toda la utilidad (o muy cercano al total con el 90\%) con el beneficio, es igual a no hacerlo sin el beneficio.

\section{DISCUSIÓN, CONCLUSIONES}

Luego de haber realizado el presente estudio, es posible determinar lo siguiente:

Existen evidentes diferencias en los efectos en la CTT entre los contribuyentes 14A y 14B dados por la tasa, por la devolución del 35\% del crédito por IRPC contra el IGC y por la injustificada 
devolución que obliga el legislador a realizar, del 50\% de los retiros efectivos que realizan estos contribuyentes, en los años siguientes de haber impetrado el beneficio en estudio. La suma de estos conceptos nos permite afirmar que los contribuyentes 14B enfrentan una mayor CTT que los contribuyentes 14A, considerando los factores y / o variables que se analizaron en este estudio (dejando de lado el hecho de cómo afectan la CTT el nivel de retiros, hecho que no se evaluó).

Si bien el objetivo que tuvo el legislador fue el "incentivo al ahorro para medianas empresas", existe un evidente privilegio al régimen 14 A por sobre los $14 \mathrm{~B}$, siendo que el nacimiento de estos últimos se debió a la necesidad de respetar la decisión de los contribuyentes de no realizar retiros, situación que no se considera para los contribuyentes del régimen de renta atribuida.

Las variables que determinan una mayor o menor CTT para los contribuyentes 14 B corresponden al nivel de retiros, siendo provechoso para ellos, en relación con ocupar o no ocupar el beneficio en estudio, mantener un nivel de retiros bajo, no superando en ningún caso el 50\%. Es más, mantener una política de retiros del $60 \%$ puede llevar a ser totalmente ineficiente el ocupar el beneficio en estudio.

Dado el nivel de ingresos que deben tener los contribuyentes que pueden beneficiarse con la disposición en estudio, el cual los categoriza como pyme, de ninguna manera puede ser más beneficioso un $14 \mathrm{~B}$ que un $14 \mathrm{~A}$ en esta materia.

Resulta paradójico que el legislador haya inspirado la disposición contenida en la letra C del artículo 4 ter de la ley del ramo en la teoría económica para establecer un "beneficio" que, de alguna u otra forma, privilegiara a un sector de la economía, pero que esta investigación determinara que genera un atentado a la igualdad horizontal, favoreciendo a empresas con igual patrimonio y características, pero que soberana o legalmente (por cumplir o no cumplir los requisitos legales) han acogido a la letra A o B del artículo 14 de la LIR y que redunde en una diferente carga tributaria total. En otras palabras, el legislador ha favorecido con un "beneficio tributario" a un tipo de contribuyentes y ha desfavorecido a otro, sin existir alguna razón técnica al respecto que, por lo menos, surja de esta investigación.

Como conclusión final a la presente investigación, es posible afirmar que el legislador ha creado dos regímenes para los contribuyentes del IRPC que son pymes, los cuales no son indistintamente provechosos para los contribuyentes, representando una mayor CTT para aquellos que son $14 \mathrm{~B}$ que los acogidos a las disposiciones $14 \mathrm{~A}$.

\section{REFERENCIAS BIBLIOGRAFICAS}

Agostini, C (2017) Reforma a la Reforma Tributaria columna de opinión publicada en El Pulso, el día 12 de abril de 2017. Obtenido de http:/ / www.pulso.cl/opinion/ reforma-la-reformatributaria

Atkinson, T., Bourguignon, F., O’Donoghue, C., Sutherland, H., \& Utili, F. (2002). Microsimulation of social policy in the European Union: Case study of a European minimum pension. Economica, 69(274), 229-243 The London School of Economics and Political Scienc.

Crocker, J. \& Slemrd, J. (2005) Corporate Tax Evasion with Cost Agency. Journal of Public Economic. 
Obtenido de: http: / / www.sciencedirect.com/ science/ article/ pii / 50047272704001537

Devereux, M y Griffith, R (2002) The Impact of Corporate Taxation on the Location of Capital: A Review. Swedish Economic Policy Review. 9 (2002) 79-102. Obtenido de: http: / / spot.colorado.edu/ utar/DevereuxGriffith.pdf

Duncan, A. (2002). Respuestas del empleo a cambios en la política fiscal obtenidas mediante microsimulación. In Conference Paper, Institute of Fiscal Studies, Madrid.

Faúndez, A y Blanco, M (2017) El Régimen de Tributación Integrado Chileno para la Microempresa y Pequeña Empresa: Desde la Perespectiva de la Equidad Tributaria Economic Analysis of Law Review. Pp 143-170. Accedido desde: https:/ / portalrevistas.ucb.br/index.php/ EALR/article/view/8152/5173

Gil, L (2007) Análisis de los sistemas de integración IRPF-IS para evitar la doble imposición de dividendos. Presentación en XIV Congreso de Economía Pública. Santander

Graham, J; Hanlon, M y Shevlin, T (2011) Real Effects of Accounting Rules: Evidence from Multinational Firms Investment Location and Profit Repatriation Decisions. Journal of Accounting Research. Vol 49 Issue 1. Pág 137-185 March 2011. Obtenido de: http: / / onlinelibrary.wiley. com/doi/10.1111/j.1475-679X.2010.00395.x/full

Hanlon, D y Pinder, S (2007) An Empirical Investigation of Whether Australian Capital Gains Tax Reforms Influencie Individual Investor Behavior Pacific Basin Finance Journal 15(2007) 481493. Accedida el 17 de Julio de 2014 http:/ / www.sciencedirect.com/science/article/pii / S0927538X0700011X

Haufler, A. (1997) Factor Taxation, Income Distribution and Capital Market Integration. Scand. Journal of Economic 99(3), 425-446. Obtenido de: http://onlinelibrary.wiley.com/ doi / 10.1111/1467-9442.00072/ full

Hernández, R; Fernández, C y Baptista, P (2010) Metodología de la Investigación. Quinta Edición. Mc Graw Hill México.

Holmes, S y Sunstein, C (2011) El Costo de los Derechos. Por qué la Libertad Depende de los Impuestos. Traducción al castellano. Título original The Cost of Rights. Bueno Aires: Siglo Veintiuno Editores. http: / / papers.ssrn.com / sol3 / paper.taf?ABSTRACT_ID=94036

Jacobs, B \& Bovenberg, A.L. (2009) Human Capital and Optimal Prositive Taxation of Capital Income. Tax Public Finance (2010) 17:451-478 DOI: 10.1007/ s10797-009-9120-5. Obtenido de: http: / / www.springerlink.com/content/a77215x2u7458167/

Larraín, F. (2011) Macroeconomía para Todos. Santiago: Editorial Pearson Educación de Chile S.A.

Mardones, C (2010) Evaluando Reformas Tributarias en Chile con un Modelo CGE. Estudios de Economía Vol 37 N² Diciembre 2010. Pag. 243-284m accedido el 16 de abril de 2017 en http: / / www.scielo.cl/ scielo.php?pid=S0718-52862010000200005\&script=sci_arttext.

Musgrave, R y Musgrave, P (1992) Hacienda Pública, Teórica y Práctica. Quinta Edición. España. Mc Graw Hill.

Otorna, G; Ottone, S; Ponzano, F y Scacciati, F. (2008) Labour Supply in Presence of Taxation Financing Public Services. An Experimental Approach. Journal of Economic Psychology 29 (2008) 619 - 631. Obtenido de: http://www.sciencedirect.com/science/article/pii/ S0167487007000578

Samuelson, P (1966) Curso de Economía Moderna, una Descripción Analítica de la Realidad Económica. Editorial Aguilar S.A. Décima quinta edición. Madrid, España.

Tarifa, E. (2005) Teoría de Modelos y Simulación. Introducción a la simulación. En doc. Virtual, Facultad de Ingeniería - Universidad Nacional de Jujuy. Accedido el 16 de abril de 2017 en http: / / www.modeladoeningenieria.edu.ar/unj/tms/apuntes/ cp1.pdf 
Taylor, S.J, y Bogdan. R (1987) Introducción a los métodos cualitativos de investigación La búsqueda de significados https: / / s3.amazonaws.com/academia.edu.documents/39626073/ Taylor-Bogdan_observacion_part.pdf?AWSAccessKeyId=AKIAIWOWYYGZ2Y53UL3A\& Expires $=1531093047 \&$ Signature $=$ Cv8Ta1 Yxy8PAIXU4pUTiswN3OLo\%3D\&response-cont ent-disposition=inline $\% 3 \mathrm{~B} \% 20$ filename\%3DTaylor_Bogdan_observacion_part.pdf 